ISSN 0103-5150

Fisioter. Mov., Curitiba, v. 25, n. 3, p. 497-506, jul./set. 2012 Licenciado sob uma Licença Creative Commons

\title{
Desafios no desenvolvimento de prontuários eletrônicos baseados em arquétipos: avaliação fisioterapêutica funcional
}

\author{
Challenges in archetype-based development of electronic \\ health records: physical therapy functional evaluation
}

\section{Daiane Cristina Martins Ronchi ${ }^{[a]}$, Dandara Novakowski Spigolon ${ }^{[b]}$, Diego Garcia ${ }^{[c]}$, Paulo Eduardo S. L. Cicogna ${ }^{[\mathrm{d}]}$, Hugo Bulegon ${ }^{[\mathrm{e}]}$, Claudia Maria Cabral Moro ${ }^{[\mathrm{f}]}$}

[a] Fisioterapeuta, especialista em Fisioterapia Neurofuncional pela Universidade Positivo (UP), mestre pelo Programa de Pós-Graduação em Tecnologia em Saúde da Pontifícia Universidade Católica do Paraná (PUCPR), professora do curso de Fisioterapia da Faculdade Educacional de Araucária (FACEAR), Curitiba, PR - Brasil, e-mail: dai_cmartins@hotmail.com

[b] Enfermeira, mestre pelo Programa de Pós-Graduação em Tecnologia em Saúde da Pontifícia Universidade Católica do Paraná (PUCPR), Curitiba, PR - Brasil, e-mail: dandaraspigolon@gmail.com

[c] Bacharel em Ciência da Computação pela Universidade do Extremo Sul Catarinense (UNESC), Criciúma, SC - Brasil, mestrando do Programa de Pós-Graduação em Tecnologia em Saúde da Pontifícia Universidade Católica do Paraná (PUCPR), Curitiba, PR - Brasil, e-mail: diegogarcia1988@gmail.com

[d] Médico pela Universidade Federal de Santa Catarina (UFSC), mestrando do Programa de Pós-Graduação em Tecnologia em Saúde da Pontifícia Universidade Católica do Paraná (PUCPR), professor do curso de Medicina da Universidade da Região de Joinville (UNIVILLE), Joinville, SC - Brasil, e-mail: pcicogna@gmail.com

[e] Bacharel em Informática, mestre pelo programa de Pós-Graduação em Tecnologia em Saúde da Pontifícia Universidade Católica do Paraná (PUCPR), Curitiba, PR - Brasil, e-mail: hugobulegon@gmail.com

[f] Doutora em Engenharia Elétrica - Engenharia Biomédica, na área de Informática em Saúde, pela Escola Politécnica da Universidade de São Paulo (USP), professora titular do Programa de Pós-Graduação em Tecnologia em Saúde da Pontifícia Universidade Católica do Paraná (PUCPR) Curitiba, PR - Brasil, e-mail: c.moro@pucpr.br

\section{Resumo}

Introdução: Cada vez mais, são necessários prontuários eletrônicos de pacientes (PEP) que contemplem informações específicas para a fisioterapia e que atendam aos requisitos de padronização e comunicação de dados. A modelagem baseada em arquétipos pode permitir a construção de PEP que atendem às necessidades da assistência ao paciente, garantindo a interoperabilidade entre sistemas e a sua usabilidade. Nesse contexto, a criação dos arquétipos é um passo crucial e determinante para um PEP específico e adequado 
para avaliação fisioterapêutica dos pacientes com lesão medular espinhal (LME). 0 objetivo deste estudo foi criar arquétipos e representá-los a partir da definição de um conjunto de dados clínicos para avaliação fisioterapêutica funcional de pacientes com LME, descrevendo os desafios, as dificuldades e as perspectivas durante o desenvolvimento e a modelagem do sistema. Método: Estudo exploratório, qualitativo e parcialmente observacional, dividido em duas fases: definição e validação dos dados clínicos para avaliação fisioterapêutica funcional de pacientes com LME e desenvolvimento dos arquétipos a partir dos dados definidos, utilizando a ferramenta OpenEHR Archetype Editor. Resultados: Foram criados 24 arquétipos a partir da elaboração de um conjunto de dados para avaliação fisioterapêutica funcional de pacientes com LME. Em seguida, foi elaborado um protótipo a partir do arquétipo "Mobilidade" da parte "Avaliação Motora", para visualizar a interface do PEP. Conclusão: O conjunto formado serviu como base essencial para criação dos arquétipos. Esta abordagem favorece uma avaliação funcional com dados relevantes e permite uma avaliação completa, adequada e com facilidade de acesso aos dados.

Palavras-chave: Avaliação em saúde. Coleta de dados. Registros eletrônicos de saúde. Sistemas de informação. Traumatismos da medula espinhal.

\section{Abstract}

Introduction: Electronic health records (EHR) that contemplate specific information for physiotherapy and cater to the required standard and data communication are more necessary each day. Archetype based modeling may allow the construction of EHR that attempts the patient's assistance needs, ensuring the interoperability between systems and its usability. In these circumstances, the archetype creation is a crucial and determinant step for a specific and adequate EHR for physical therapy evaluation of patients with spinal cord injury (SCI). The aim of this study was to create archetypes and represent through the definition of a clinical data set for evaluation of patients with SCI, describing the challenges, the difficulties and the perspectives during the system's development and the modeling. Methods: Exploratory study, divided in two phases: definition and validation of clinical data set for physical therapy functional evaluation of patients with SCI and archetype development based on the data defined, using the OpenEHR Archetype Editor. Results: 24 archetypes were created after the development of a data set for physical therapy functional evaluation of patients with SCI. After that, a prototype was developed after the archetype "Mobility" of the "Motor Evaluation" part to visualize the EHR interface. Conclusion: The data set developed was essential for the archetype's creation. This approach facilitates a functional evaluation with relevant data, and allows a complete and adequate evaluation with easy access to the data.

Keywords: Health evaluation. Data collection. Electronic health records. Information systems. Spinal cord injuries.

\section{Introdução}

São raros os Prontuários Eletrônicos do Paciente (PEP) disponíveis para a área de fisioterapia. Isso dificulta a atuação do fisioterapeuta, uma vez que ele não conta com dados específicos relacionados aos atendimentos que realiza. Atualmente, existem algumas iniciativas de propostas de sistemas e de dados para a fisioterapia, como o PEP apresentado por Buyl e Nyssen (1). Porém, nessa proposta, a fisioterapia foi abordada de forma geral, não contemplando os dados necessários para os atendimentos relacionados às áreas específicas, como: avaliação fisioterapêutica, fibromialgia, desenvolvimento neuropsicomotor, entre outros.

Para o desenvolvimento de um PEP estruturado e organizado de forma eficiente, é importante considerar os padrões de saúde. Dentre eles, destaca-se a modelagem de sistemas baseada em arquétipos. Esse padrão foi proposto pela Fundação OpenEHR (http://www.openehr.org), sendo considerado eficaz no desenvolvimento de PEP interoperáveis, possibilitando a representação semântica das informações e dos dados clínicos (2). A interoperabilidade semântica entre PEP é fundamental para a integração entre as informações dos diferentes 
sistemas, necessária para a existência de Registros Eletrônicos em Saúde (RES).

Considerando-se essas questões, o Ministério da Saúde do Brasil regulamentou o uso de padrões de interoperabilidade e informação em saúde para o RES na portaria $\mathrm{n}^{\circ}$. 2.073, de 31 de agosto de 2011, determinando que sejam utilizados os arquétipos. $\mathrm{Na}$ definição do RES nacional, será utilizado o modelo de referência definido pela OpenEHR. Para a interoperabilidade de modelos de conhecimento, incluindo arquétipos, templates e metodologia de gestão, será utilizado o padrão ISO 13606-2 (3).

As informações presentes em um arquétipo devem ser suficientes para serem interpretadas isoladamente e tão completas quanto possível para atender múltiplos setores, propósitos e prioridades (4). 0 conceito clínico pode ser representado formalmente pelos especialistas do domínio (profissionais da saúde) e reutilizado, facilitando o compartilhamento do conhecimento.

Nesse contexto, a criação de arquétipos favorece a padronização do PEP e deve ser considerada na especificação de todos os sistemas de informação em saúde (SIS). Sabe-se que a avaliação fisioterapêutica dos pacientes é essencial em diversas áreas. Uma delas, que merece especial atenção em virtude de sua condição clínica, incapacitante, aguda e imprevista, é o atendimento aos pacientes com lesão medular espinhal (LME). Considerando-se que não existe um conjunto de dados específico para registro da avaliação fisioterapêutica de pacientes com LME, faz-se necessário especificar um conjunto de dados representado por arquétipos.

0 atendimento do fisioterapeuta é de extrema importância na LME e justifica-se por essa condição afetar as funções motoras, sensoriais e autonômicas do paciente, alterando a vida do indivíduo acometido e de sua família e gerando desastrosas consequências físicas, psicológicas, sociais e econômicas (5-7). É a maior causa de morbimortalidade entre adultos jovens com idade entre 18 e 40 anos, predominantemente no sexo masculino, na proporção de quatro homens para cada mulher $(4: 1)(6,8,9)$.

No Brasil, os únicos dados padronizados que os fisioterapeutas utilizam para o atendimento da LME são as escalas para avaliação e acompanhamento desses pacientes, como a Escala de Classificação Neurológica da Lesão Medular, a Medida de Independência Funcional (MIF), o Índice de Barthel (IB), a Berg Balance Scale (BBS) e o Walking Index for Spinal Cord Injury II (WISCI II) $(5,10)$. Porém, as escalas são instrumentos de avaliação rápida, realizada em papel, em forma de checagem e pontuação, sujeita a erros de interpretação e sem garantia de armazenamento seguro dos resultados das informações do paciente.

Apesar disso, não há nada em relação ao PEP para avaliação e acompanhamento de pacientes com LME, de forma que se tenham os registros dos eventos clínicos referentes a cada indivíduo de maneira padronizada, estruturada e que forneça informações necessárias para o acompanhamento pela equipe de saúde que realiza os atendimentos.

É importante ressaltar que, para definir um PEP com dados representados em arquétipos, existem desafios durante esse processo, como: a própria elaboração dos arquétipos; a forma de representar os dados; os tipos de elemento e de estrutura a utilizar; as ferramentas que estão disponíveis para sua formação e aplicação; a escolha do modelo de referência; e outros, que serão apresentadas no decorrer do estudo.

Considerando-se a necessidade de PEP para a fisioterapia e a importância de baseá-lo em padrões da área de saúde, objetivou-se elaborar arquétipos e representá-los a partir de um conjunto de dados para avaliação fisioterapêutica funcional de indivíduos com LME, descrevendo os desafios, as dificuldades e as perspectivas durante o processo de desenvolvimento e modelagem do sistema.

\section{Métodos}

Trata-se de um estudo com característica exploratória qualitativa (11), parcialmente observacional, dividido em duas fases: definição e validação do conjunto de dados; e desenvolvimento dos arquétipos.

Fase I - Definição e validação do conjunto de dados

O levantamento e a verificação dos dados necessários para avaliação do paciente com LME e das necessidades do PEP, incluindo a análise das características de usabilidade, foi realizado com base em uma revisão de literatura e complementado com os dados coletados nas observações de atendimentos dos fisioterapeutas, em uma instituição que atende pacientes com LME em fase de reabilitação. 
As bases de dados utilizadas foram: SciELO (http:// www.scielo.br), LILACS (http://www.bireme.com.br) e PubMed (http://www.pubmed.com.br), utilizando as seguintes palavras-chave: lesão medular espinhal; traumatismo raquimedular; avaliação fisioterapêutica funcional; escalas de avaliação funcional; reabilitação; sistemas de informação em saúde; prontuários eletrônicos do paciente; e arquétipos.

Foram identificadas na internet as instituições que atendem pacientes com LME em fase de reabilitação, selecionando aquelas que se enquadravam nos seguintes critérios de inclusão: realizar atendimento de pacientes com LME no primeiro ano de lesão; ter fisioterapeutas especialistas em neurologia funcional; e permitir o acompanhamento dos atendimentos. A partir disso, foi definido o Centro de Reabilitação Hospitalar Ana Carolina Moura Xavier (CHR), de Curitiba, PR, como local de estudo observacional.

Baseando-se nos dados encontrados na literatura, na observação e no acompanhamento da rotina de trabalho dos fisioterapeutas do CHR foi formado um conjunto de dados essenciais para avaliação do paciente com LME.

Para a validação por especialistas, esses dados foram estruturados em um questionário denominado "Dados essenciais para avaliação fisioterapêutica funcional de pacientes com lesão medular", que ficou disponível no GoogleDocs.

Os dados do questionário foram divididos em três partes, e cada parte foi dividida em categorias.

Na parte I, consta o perfil do participante, com dados como: profissão, nível de formação, pós-graduação e tempo de atuação com pacientes com LME.

Na parte II, encontram-se dados pessoais, dados clínicos, histórico da lesão e anamnese, no qual o profissional foi orientado a selecionar apenas os dados que julgasse essenciais para a avaliação fisioterapêutica funcional do paciente com LME.

Na parte III, a avaliação fisioterapêutica foi categorizada em sistemas, como: motor, sensorial, pele, pulmonar, cardiovascular, urodinâmico e intestinal, além de qualidade de vida, escalas de avaliação funcional e conduta do profissional.

Além dos profissionais do CHR, também foram identificados, para responder ao questionário, fisioterapeutas especialistas em neurologia que trabalham em instituições de reabilitação no Brasil e que têm publicações na área de lesão medular, por meio de busca do Currículo Lattes (http://lattes.cnpq.br/).
O questionário foi enviado por e-mail a 28 fisioterapeutas, de instituições de reabilitação no Brasil que atendem pacientes com LME, tendo como prazo para retorno 30 dias.

Os profissionais foram orientados a responder à parte III do questionário indicando o nível de importância de cada dado coletado, baseando-se na Escala de Importância de Likert, que varia de "Extrema importância" (5), "Muito importante" (4) e "Importante" (3) a "Pouco importante" (2) e "Sem importância" (1). Nas outras duas partes do questionário, a resposta era direta.

Os fisioterapeutas que participaram dessa pesquisa, assim como os pacientes, cujos atendimentos foram observados, receberam esclarecimentos quanto ao propósito do estudo e assinaram um Termo de Consentimento Livre e Esclarecido. A pesquisa foi aprovada pelo Comitê de Ética em Pesquisa da Pontifícia Universidade Católica do Paraná (PUCPR), sob o parecer n. 0004962/11, e pelo Comitê de Ética em Pesquisa da Secretaria de Estado de Saúde do Paraná (SESA-PR), sob o parecer n. 346/2011.

Foram respondidos 12 questionários, e a análise das respostas foi realizada em cada uma das três partes, separadamente.

Parte I - resultados do perfil do participante. Foram considerados o número de profissionais que são especialistas em neurologia e o tempo de atuação como profissional com pacientes com LME.

Parte II - resultados de dados pessoais, dados clínicos, histórico da lesão e anamnese. Foram considerados, para inclusão no PEP, os dados selecionados como essenciais para a avaliação fisioterapêutica funcional de pacientes com LME, por mais de $60 \%$ dos profissionais participantes.

Parte III - resultados da avaliação fisioterapêutica. Foram selecionados os dados indicados pela maioria dos participantes (> 60\%) como extrema importância, muito importante ou importante. Em caso de dúvida em relação à resposta dos profissionais, foram considerados os dados utilizados pelos fisioterapeutas na observação da avaliação, da reavaliação e dos atendimentos no CHR com uma frequência igual ou maior a 70\%. Na persistência da dúvida, foi analisado o periódico de publicação do artigo do qual o dado foi coletado. Para o dado ser incluído no conjunto final, o periódico deveria ter avaliação no Qualis da CAPES superior a B2, nas áreas de Educação Física, Medicina e Interdisciplinar, ou fator de impacto superior a 1,5. 
Fase II - Desenvolvimento dos arquétipos

A criação dos arquétipos foi feita a partir da definição dos dados clínicos para avaliação fisioterapêutica funcional do paciente com LME.

Primeiramente, foi escolhido o editor LinkEHR- ed para a criação dos arquétipos, pois a maioria dos estudos encontrados utilizou essa ferramenta. Em seguida, foi realizada uma avaliação dos elementos, dados que poderiam ser utilizados na elaboração dos arquétipos. A "Mobilidade" foi o conceito clínico escolhido para ser modelado, servindo de exemplo para a criação dos demais arquétipos.

Tanto o padrão aberto OpenEHR quanto a norma ISO 13606 utilizam a linguagem Archetype Definition Language (ADL) para a construção dos arquétipos, porém existem algumas diferenças entre eles. A ISO 13606 tem como foco primário a comunicação entre diferentes RES e define o extrato do RES, enquanto o OpenEHR visa definir uma arquitetura padronizada para a representação de informações em saúde e inclui sistemas no seu escopo. Além disso, o modelo de referência da ISO 13606 é mais simples que o modelo do OpenEHR, que possui maior especialização dos elementos e das estruturas de dados não existentes na ISO 13606, como o caso de tabelas e árvores.

0 modelo OpenEHR está baseado em uma ontologia de conceitos representados por arquétipos, organizados nas seguintes categorias (2): compositions de arquétipos temáticos - documentos clínicos como: sumário de alta, avaliação pré-anestésica, cuidados de enfermagem, entre outros; sections - arquétipos organizacionais utilizados para definir a navegação no RES: história, exame clínico e evoluções; observations - registro de dados mensuráveis ou observados: pressão arterial, sintomas, peso; evaluations - registro de avaliações clínicas: avaliação de risco anestésico, efeito adverso; instructions - registro no início de processo de workflow: prescrição médica, solicitação de exames; actions - registro da atividade clínica: administração de medicamentos ou realização de procedimentos. Actions complementam as instructions e registram seus estados, tais como: completed ou cancelled.

Para definição dos elementos, foram utilizadas as entidades evaluation e observation. Para o agrupamento dos elementos, foram utilizados inicialmente sections; porém, considerando-se que elas não estão disponíveis em todas as ferramentas de edição dos arquétipos, optou-se então pela estruturação em clusters. Eles têm a mesma finalidade das sections e possibilitam a utilização de diferentes editores.

Ao elaborar um elemento que possui uma lista de valores possíveis, verificou-se que a ferramenta LinkEHR-ed não apresenta uma forma clara de definição. Por exemplo, para determinar o "grau de dor ao movimento", que é especificado pela Escala Analógica de Dor, é necessário possibilitar o registro de dez valores entre 0 e 10 . Nesse editor, não ficou claro quais passos deveriam ser seguidos para adicionar um elemento que possua uma lista de valores.

Verificou-se que a ferramenta OpenEHR Archetype Editor possibilita uma nova abordagem na geração desse tipo de elemento, ou seja, foi possível adicionar apenas um elemento Text, selecionar a opção Freeorcodedtext e inserir os valores. A partir disso, verificou-se que o processo de geração de arquétipo nessa ferramenta é mais simples que a ferramenta previamente selecionada. Por essa razão, foi dado continuidade à criação dos arquétipos utilizando-se o OpenEHR Archetype Editor.

Outra característica diferencial do OpenEHR Archetype Editor em relação ao LinkEHR-ed é que, durante a elaboração arquétipo, é possível visualizar sua interface e, ainda, permite que cada dado seja descrito de acordo com sua funcionalidade.

Os arquétipos foram representados utilizando-se a linguagem ADL, que fornece uma sintaxe formal e abstrata para descrever restrições de qualquer entidade de domínio, consistindo basicamente de três partes: identificação; definição - estrutura, regras e cardinalidade -; e ontologia $(12,13)$.

Para cada categoria da Parte III - Avaliação Fisioterapêutica Funcional, foi elaborado um arquétipo no OpenEHR Archetype Editor com os dados validados pelos fisioterapeutas especialistas na Fase III, iniciando pelo arquétipo "Mobilidade".

Baseando-se nos dados validados, foram elaborados 24 arquétipos para compor o Prontuário Eletrônico do Paciente com LME (PEP_REAB). Os arquétipos foram criados separadamente, para que possam ser utilizados por outros profissionais na abordagem de diferentes tipos de pacientes. Em seguida, foi elaborada uma tela, a partir do arquétipo "Mobilidade" da parte de Avaliação Motora.

Em 15 arquétipos elaborados, foi utilizada a entidade evaluation, pois o fisioterapeuta necessita examinar o paciente. Nos casos em que o profissional apenas questiona o paciente ou recebe informações advindas de encaminhamento do médico ou de outros 
profissionais, foi utilizada a entidade observation, ou seja, oito arquétipos foram desenvolvidos utilizando-se essa entidade. Apenas no arquétipo "Conduta do profissional" foi utilizada a entidade entry, pois se trata de uma declaração clínica elaborada pelo fisioterapeuta. 0 Quadro 1 ilustra as entidades escolhidas para a criação de cada arquétipo.

A parte "Mobilidade" da Avaliação Motora foi modelada como uma evaluation, com a estrutura de uma tree (árvore), na qual cada um dos três itens (restrição de movimento, contratura muscular e dor ao movimento) da avaliação de mobilidade foi definido como cluster. Cada cluster contém seus elements. Por exemplo, no cluster "dor ao movimento" há três elements: dor ao movimento, cuja resposta é "sim" ou "não"; local, que se trata de um texto livre para designar o local da dor; e grau de dor, que utiliza a Escala Analógica de Dor como forma de mensuração.

\section{Quadro 1 - Entidades dos arquétipos}

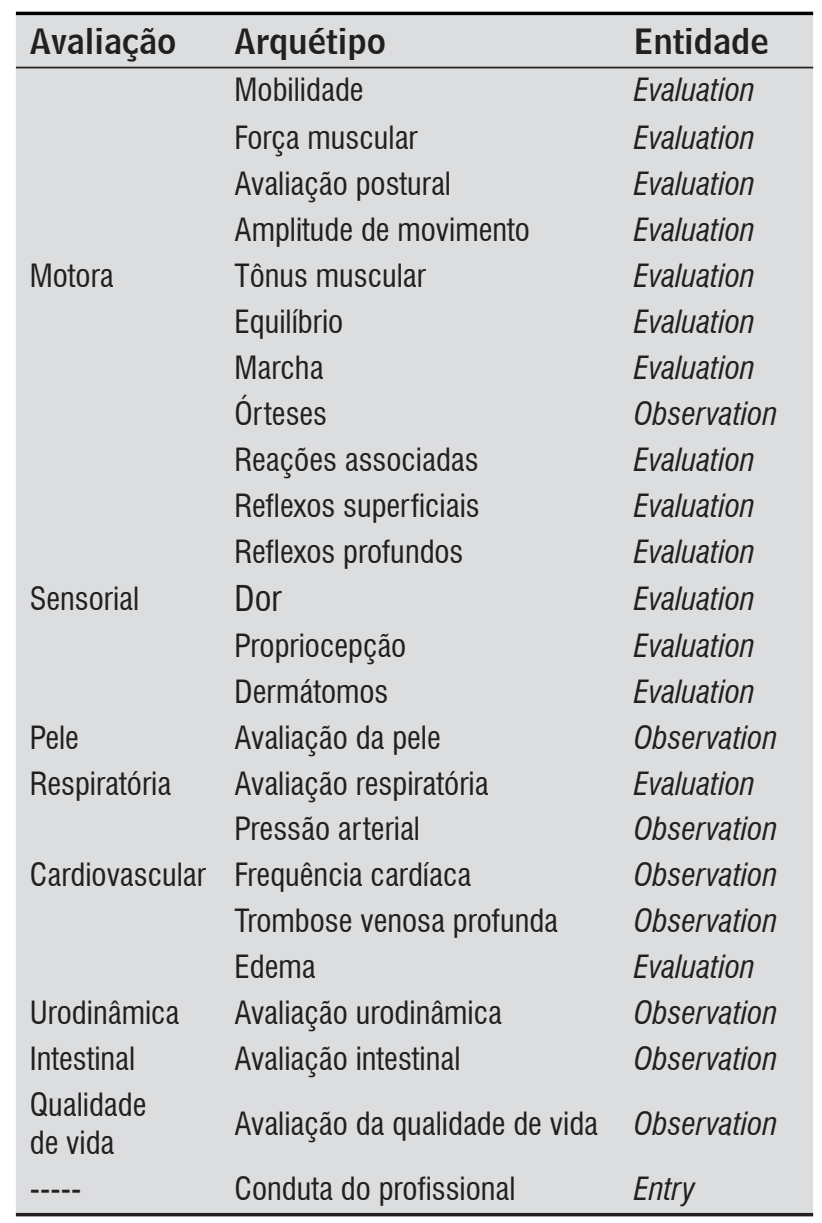

Fonte: Dados da pesquisa.
Foi desenvolvido um protótipo de interface para o arquétipo "Mobilidade", utilizando a abordagem manual, para cujo desenvolvimento o arquétipo é utilizado como base. Nessa abordagem, as alterações feitas no arquétipo não são refletidas automaticamente pela interface, sendo nesse caso necessária a intervenção do desenvolvedor.

O PEP elaborado a partir dos arquétipos criados foi construído com interface web utilizando o Java Server Faces 2.0 (JSF2), com a biblioteca PrimeFaces 3.0. 0 ambiente de desenvolvimento utilizado foi Eclipse Helios e, para a execução da aplicação, o servidor de aplicações JBoss Application Server 7. A Figura 1 apresenta a interface do PEP criada com base no conjunto de dados referente à avaliação da mobilidade.

\section{Resultados}

Foram desenvolvidos 24 arquétipos, de forma que possam ser utilizados em PEP de diferentes áreas da saúde e com pacientes com patologias distintas. A Figura 2 ilustra o arquétipo "Mobilidade", que é do tipo evaluation e foi elaborado utilizando-se o OpenEHR Archetype Editor. A terminologia e a interface desse arquétipo estão representadas pelas Figuras 3 e 4, respectivamente.

\section{Discussão}

A crescente necessidade de prontuários que contemplem informações específicas para a fisioterapia e que atendam aos requisitos de padronização de dados e comunicação faz com que estudos, como o apresentado neste trabalho, sejam essenciais - em especial, nos casos cujos conjuntos de dados não estão definidos, como na avaliação de pacientes com LME. Essa é uma patologia que precisa de dados específicos para o atendimento de pacientes, considerando-se principalmente o número de indivíduos que podem ser beneficiados com essas informações. No Brasil, existem 130 mil indivíduos portadores dessa patologia, em função do aumento anual decorrente de acidentes automobilísticos e da violência urbana (14).

0 cuidado do fisioterapeuta pode trazer uma contribuição significativa para esses pacientes. Porém, a forma como esses profissionais vão conduzir seu atendimento e tomar decisões importantes depende 


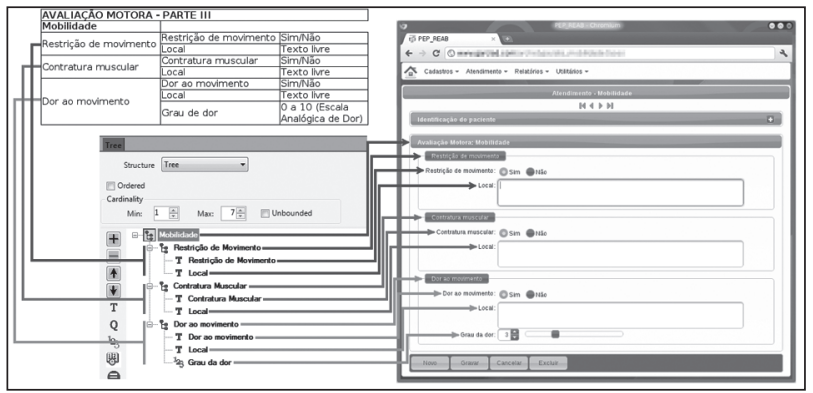

Figura 1 - Interface para 0 arquétipo "Mobilidade" Fonte: Dados da pesquisa.

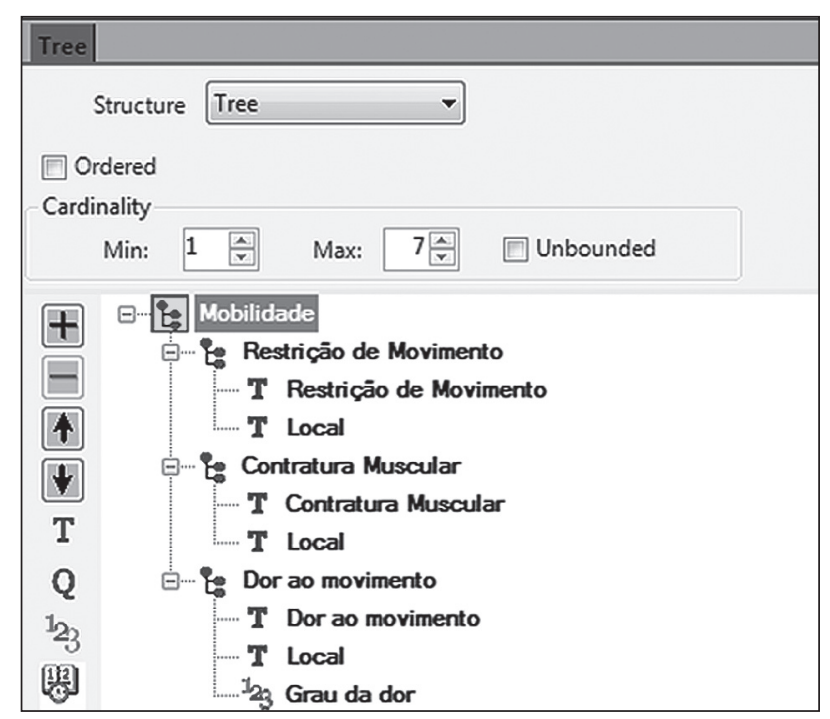

Figura 2 - Arquétipo "Mobilidade"

Fonte: Dados da pesquisa.

de uma série de fatores, dentre eles, o acesso à informação necessária e adequada (1).

A informatização do prontuário é essencial para atender às necessidades de acesso às informações, pois o PEP adequado traz diversos benefícios, como: dados estruturados; melhora na qualidade da informação disponível; agilidade do acesso aos registros; compartilhamento de informações sobre o paciente e acesso simultâneo a elas. Ou seja, todas as informações do paciente estão disponíveis de forma organizada e com possibilidade de acesso rápido. Isso facilita, também, a integração e a comunicação da equipe de saúde durante o atendimento $(1,15,16)$.

Porém, é importante ressaltar que as informações devem ser definidas não somente para um PEP específico, mas sim da maneira mais abrangente possível e que facilite a troca de dados entre diferentes sistemas.

\begin{tabular}{|c|c|c|}
\hline \multicolumn{3}{|c|}{ Archetype Editor [pt-br] Mobilidade } \\
\hline \multicolumn{3}{|c|}{ e Edit Language Terminology Display Tools Help } \\
\hline \multicolumn{3}{|l|}{ 回 } \\
\hline \multicolumn{3}{|c|}{ penEHR-EHR-EVALUATION.mobilidade.v1 } \\
\hline \multicolumn{3}{|c|}{ 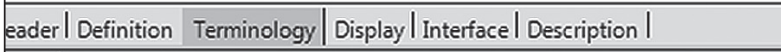 } \\
\hline \multicolumn{3}{|c|}{\begin{tabular}{|l|l|} 
rms & Bindings I Constraints I Languages_Terminologies I
\end{tabular}} \\
\hline \multicolumn{3}{|l|}{ rm definitions } \\
\hline Code & Text & Description \\
\hline at0000 & Mobilidade & Mobilidade \\
\hline at0002 & Dor ao movimento & This is a CLUSTER object \\
\hline at0003 & Contratura Muscular & Contratura muscular \\
\hline at0004 & Tree & This is a MEM_TREE object \\
\hline at0005 & Mobilidade & This is a CLUSTER object \\
\hline at0006 & Contratura Muscular & Contratura muscular \\
\hline at0009 & Restrição de Movimento & This is a CLUSTER object \\
\hline at0010 & Restrição de Movimento & Restri??o de movimento \\
\hline at0012 & Dor ao movimento & This is a ELEMENT object \\
\hline at0013 & Local & This is a ELEMENT object \\
\hline at0016 & Local & Local \\
\hline at0025 & Local & This is a ELEMENT object \\
\hline at0029 & Grau da dor & Grau da dor \\
\hline at0043 & Sim & Sim \\
\hline at0044 & Não & Não \\
\hline at0045 & Sim & Sim \\
\hline at 0046 & Não & Não \\
\hline at 0047 & Sim & Sim \\
\hline at0048 & Não & Não \\
\hline
\end{tabular}

Figura 3 - Terminologia do arquétipo "Mobilidade" Fonte: Dados da pesquisa.

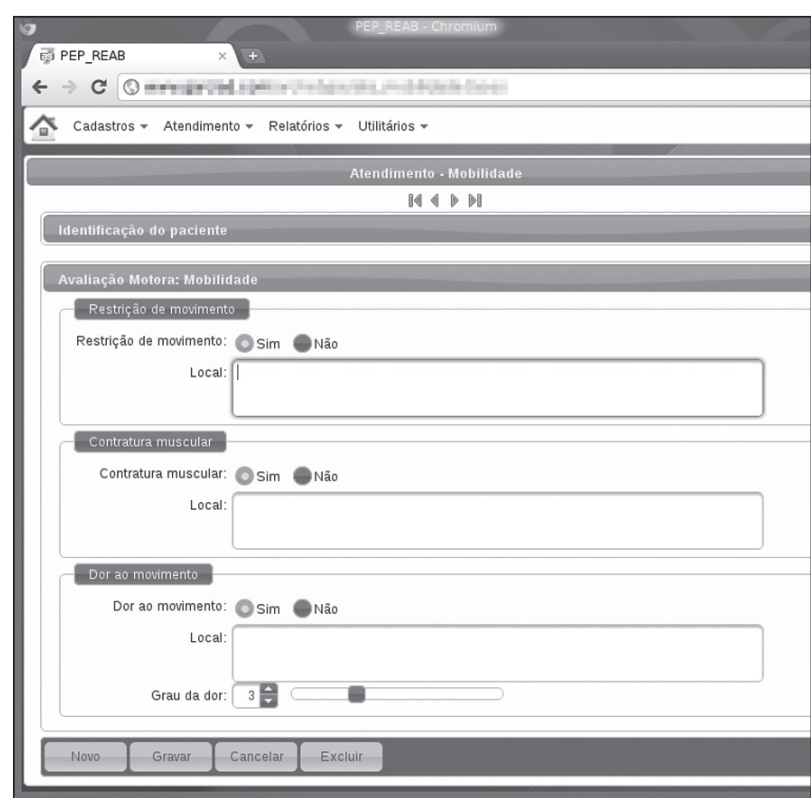

Figura 4 - Protótipo elaborado a partir do arquétipo "Mobilidade"

Fonte: Dados da pesquisa. 
Por esses motivos, optou-se pela validação do conjunto de dados pelos especialistas e a modelagem baseada em arquétipos. Muitas vezes, os sistemas são definidos com uma visão bem específica, como o apresentado por Buyl e Nyssen (1). Os arquétipos permitem que esse conjunto de dados possa ser utilizado em diversos PEP e, ainda, facilitam a troca de informações entre sistemas.

Como os fisioterapeutas buscam a padronização dos dados para favorecer seus serviços e os arquétipos têm como característica os padrões em saúde, eles podem ser eficazes na criação de fichas clínicas de forma interoperável e semântica, em qualquer domínio. Esse padrão de modelagem pode permitir a comunicação entre os registros de forma significativa nas condutas dos profissionais, além de auxiliar o desenvolvimento do sistema $(2,17,18)$.

Durante o processo de desenvolvimento dos arquétipos, foi possível comprovar algumas vantagens citadas na literatura, dentre elas: a informação clínica pode ser criada e modificada a qualquer momento, sem afetar o modelo de objeto do software ou da estrutura do banco de dados; permite definir um conhecimento comum partilhado por todos os atores envolvidos no processo de atendimento; o acesso aos dados pode ser controlado; permite utilizar a base de conhecimento para processamento automático, como sistemas de apoio à decisão; e permite a definição e o controle do conhecimento em saúde em nível dos conceitos (19).

Por outro lado, suas desvantagens estão associadas a: dificuldades encontradas durante seu desenvolvimento; algumas estruturas de dados que não possuem informação suficiente para que sejam bem representadas em uma entidade; desafios na construção da interface gráfica; necessidade de um serviço de terminologia que não perca sua portabilidade semântica; custos para a equipe técnica no desenvolvimento de templates, pois não existem editores gratuitos (20).

Neste estudo, o principal desafio esteve relacionado à fase inicial do desenvolvimento dos arquétipos, na caracterização entre as diferentes entidades disponíveis. Na literatura consultada, não está claro qual entidade era utilizada para os conceitos que foram selecionados no início. Foi identificada uma restrição quanto ao uso de determinados elementos na ferramenta OpenEHR Archetype Editor, que impedia a visualização de arquétipos desenvolvidos na ferramenta LinkEHR-ed, apesar de o elemento em questão - section - fazer parte da especificação do modelo de referência. A definição de uma lista de valores possíveis para um determinado elemento também é feita de forma diferenciada entre as ferramentas. Isso faz com que os arquétipos, mesmo sendo padrões, possam não ser totalmente reaproveitados nos PEP.

Atualmente, a experiência do uso de arquétipos implementados em sistemas para registros eletrônicos em saúde é restrita a poucos exemplos $(16,17$, 20). Mesmo assim, as tendências são positivas na interoperabilidade dos RES no futuro (17).

Além disso, os modelos com conteúdo podem ser usados não só para obter formas de telas, mas, também, para facilitar a consulta de interfaces genéricas e para construir mensagens de comunicação entre diferentes sistemas de registros eletrônicos em saúde (21).

Existem experiências com o desenvolvimento de arquétipos, como as de Neira et al. (2), Dias e Freire (13) e Nardon et al. (20), as quais trazem resultados positivos na utilização dos arquétipos, com resolutividade nos objetivos que os profissionais de saúde esperam dos PEP. Porém, todos encontram desafios e dificuldades semelhantes, principalmente no entendimento da ferramenta e em sua utilização. Isso pode ocorrer em virtude dos poucos estudos que aplicam os PEP depois de definidos, além da falta de padronização de conceitos e de modelos dos dados, o que acarreta esforços múltiplos e redundantes para análise e desenvolvimento de sistemas, dificultando a interpretação no significado de cada informação. Isto é, a dificuldade maior não está em especificar o arquétipo, mas sim em utilizá-lo novamente em outros sistemas. Por isso, a especificação de conjuntos de dados validados por especialistas é fundamental para o desenvolvimento de arquétipos que atendam às necessidades de diferentes PEP.

\section{Conclusão}

O conjunto de dados clínicos essenciais que foi definido para avaliação fisioterapêutica funcional do paciente com LME serviu como base essencial para criação dos arquétipos.

A ideia é que, utilizando esse tipo de modelagem para desenvolver um PEP, as informações sejam registradas de forma organizada, eficiente, interoperável e semântica, respeitando os padrões em saúde. 
Com isso, é possível proporcionar aos fisioterapeutas uma avaliação funcional com dados relevantes para a elaboração dos objetivos do programa de tratamento e para o acompanhamento da evolução do paciente, permitindo a esses profissionais uma avaliação completa, adequada e com facilidade de acesso aos dados necessários para a abordagem ao paciente. Além disso, possibilita avaliar a eficácia do atendimento prestado, dar suporte para a alta do paciente e o encaminhamento para outras instituições.

É importante ressaltar que muitos desafios foram e ainda serão encontrados durante o processo de definição de dados, desenvolvimento e, principalmente, estruturação e aplicação dos arquétipos nos PEP. Apesar disso, acredita-se positivamente nas perspectivas que os arquétipos irão oferecer para o futuro dos PEP e, principalmente, para atender às necessidades dos profissionais de saúde.

\section{Referências}

1. Buyl R, Nyssen M. Structured electronic physiotherapy records. Int J Med Inform. 2009;78(7):473-81. doi:10.1016/j.ijmedinf.2009.02.007.

2. Neira RAQ, Nardon FB, Moura LA Jr., Leão BF. Como incorporar conhecimento aos sistemas de registro eletrônico em saúde? Anais do XI Congresso Brasileiro de Informática em Saúde - CBIS. Campos de Jordão: São Paulo; 2008.

3. Brasil. Ministério da Saúde. Portaria no 2.073, de 31 de agosto de 2011. Regulamenta o uso de padrões de interoperabilidade e informação em saúde para sistemas de informação em saúde no âmbito do Sistema Único de Saúde, nos níveis Municipal, Distrital, Estadual e Federal, e para os sistemas privados e do setor de saúde suplementar. Diário Oficial [da] União. 2011; Seção 1. p. 63 [citado 30 dez. 2011]. Disponível em: http://www. in.gov.br/imprensa/visualiza/index.jsp?jornal=1\&pagi na $=63 \&$ data $=01 / 09 / 2011$.

4. Santos MR, Bax MP. Modelagem de um repositório central baseado em arquétipos para sistema de RES federados. Anais do XII Congresso Brasileiro de Informática em Saúde - CBIS. Porto de Galinhas: Pernambuco; 2010.

5. Scivoletto G, Ditunno P, Ditunno JF, Molinari M. Walking index for spinal cord injury (WISCI): criterion validation. Spinal Cord. 2005;43(1):27-33. doi:10.1038/ sj.sc.3101658.
6. Cristante ARL. Aspectos clínicos. In: Moura EW, Silva PAC. Fisioterapia: aspectos clínicos e práticos da reabilitação. São Paulo: Artes Médicas; 2005. p. 501-2.

7. Campos MF, Ribeiro AT, Listik S, Pereira CAB, Sobrinho JÁ, Rapoport A. Epidemiologia do traumatismo da coluna vertebral. Rev Col Bras Cir. 2008;35(2):88-93.

8. Cardozo-Gonzales RI, Villa TCS, Calibri MHL. O processo da assistência ao paciente com lesão medular: gerenciamento de caso como estratégia para organização da alta hospitalar. Medicina. 2001;34(3-4):325-33.

9. Rede Sarah de Hospitais do Aparelho Locomotor. Manual do lesado medular. Brasília: Sarah Letras; 1999.

10. Schmitz TJ. Lesão medular espinhal. In: O'sullivan SB, Schmitz TJ. Fisioterapia: avaliação e tratamento. 4a ed. São Paulo: Manole; 2004. p. 207-30.

11. Tobar F, Yalour MR. Como fazer teses em saúde pública: conselhos e ideias para formular projetos e redigir teses e informes de pesquisas. Rio de Janeiro: Fiocruz; 2001.

12. Portal Público da Base de Registro Eletrônico em Saúde do Estado de Minas Gerais. Arquétipos publicados, Belo Horizonte; [citado 30 nov. 2010]. Disponível em: http:// sres.saude.mg.gov.br/arquetipo/listar.

13. Dias RDM, Freire SM. Arquétipos para representar informações demográficas em saúde. Anais do XI Congresso Brasileiro de Informática em Saúde - CBIS. Campos de Jordão: São Paulo; 2008.

14. Rodrigues D, Herrera G. Recursos fisioterapêuticos na prevenção da perda da densidade mineral óssea com lesão medular. Acta Ortop Bras. 2004;12(3):183-8. doi:10.1590/S1413-78522004000300008.

15. Häyrinen K, Saranto K, Nykänen P. Definition, structure, content, use and impacts of electronic health records: a review of the research literature. Int J Med Inform. 2008;77:291-304. doi:10.1016/j.ijmedinf.2007.09.001.

16. Mourão AD, Neves JTR. Impactos da implantação do prontuário eletrônico do paciente sobre o trabalho dos profissionais de saúde da Prefeitura Municipal de Belo Horizonte. Faculdade Cenecista de Varginha - FACECA, 2007; [citado 15 jun. 2010]. Disponível em: http://www. economia.aedb.br/seget/artigos07/56_SEGET.pdf.

17. Chen R, Klein GO, Sundvall E, Karlsson D, Ahlfeldt H. Archetype-based conversion of EHR content models: pilot experience with a regional EHR system. BMC Med Inform Decis Mak. 2009;9(33):1-13. PMid:19138407.

18. Maldonado JA, Moner D, Tomás D, Angelo C, Robles M, Fernandez JT. Framework for clinical data standardization base on archetypes. Stud Health Technol Inform. 2007;129(Pt 1):454-8. PMid:17911758. 
Ronchi DCM, Spigolon DN, Garcia D, Cicogna PESL, Bulegon H, Moro CMC.

19. Purin B, Eccher C, Forti S. A real application of a concept based electronic medical record. AMIA Annu Symp Proc. 2003:977. PMid:14728481.

20. Nardon FB, França T, Naves H. Construção de aplicações em saúde baseadas em arquétipos. Anais do XI Congresso Brasileiro de Informática em Saúde - CBIS. Campos de Jordão: São Paulo; 2008.
21. Chen R, Enberg G, Klein GO. Julius: a template based supplementary electronic health record system. BMC Med Inform Decis Mak. 2007;7(10):1-11. PMid:17214888

Recebido: 06/06/2012

Received: 06/06/2012

Aprovado: 05/08/2012

Approved: 08/05/2012 